\title{
Aorto-oesophageal fistula secondary to impaction of a fish bone at Autopsy
}

\author{
Fernando DMG, Izzath MHMA, Jayasooriya RP, Rathnayake RMIS
}

\begin{abstract}
Aorto-oesophageal fistula is a life threatening condition in which there is an abnormal connection between the aorta and oesophagus, and is a rare complication of foreign body ingestion from which few patients survive. Classic presentation is with Chiari's triad of mid thoracic pain, sentinel arterial haemorrhage and massive exsanguination after a symptom free interval. A 36-year-old male was transferred to a tertiary care hospital with a history of epigastric pain and discomfort in his retro-sternal area. According to the history, he had ingested fish the previous day while consuming alcohol. He was treated for four days in the hospital, and discharged with a diagnosis of alcohol induced gastritis. He was re-admitted later the same day with an episode of haematemesis. However, investigations such as chest radiography and oesophagogastroduodenoscopy were not carried out. He developed massive haematemesis 12 hours later, and despite vigorous resuscitation and emergency surgery, he expired. Autopsy revealed an impacted fish bone at the junction of upper and middle third of the oesophagus creating a fistula tract from oesophagus to thoracic aorta which resulted in exsanguination, haemorrhagic shock and death. Even though mortality is very high, survival is possible with a high index of suspicion, early diagnosis and rapid surgical intervention. Autopsy findings, review of the literature and medico-legal aspects of possible medical negligence are discussed.
\end{abstract}

Key words: Exsanguination; Fish bone; Medical negligence; Chiari’s triad; Impaction

\section{Full paper}

\section{Introduction}

Accidental ingestion of foreign bodies is a common problem; once ingested they may get stuck at an area of oesophageal constriction. If they do not pass, most oesophageal foreign bodies (EFB) can be retrieved by oesophagoscopy. Aorto-oesophageal fistula (AEF) which is a rare and catastrophic condition may be primary or secondary. The main predisposing factors for primary AEF is atherosclerotic thoracic aortic aneurysm and ingestion of foreign bodies. ${ }^{[1]}$ An AEF is a rare cause of upper gastrointestinal bleeding. Aorto-oesophageal fistulae following foreign body ingestion are rare, and almost always lethal, but survival is now possible with rapid surgical interventions such as thoracic endovascular aortic repair followed by oesophageal surgery. ${ }^{[1]}$ A

Department of Forensic Medicine, Faculty of Medicine, University of Peradeniya, Sri Lanka

Corresponding author: Fernando DMG, email: dineshmgfdo@yahoo.com

DOI: http://doi.org/10.4038/mljsl.v4i2.7340 high index of suspicion is needed for early diagnosis, which might improve the chance of survival.

\section{Case report}

A 36 years old healthy unemployed alcoholic male presented to a district hospital complaining ofepigastric pain and feeling of something sticking in the retrosternal area following ingestion of fish with arrack(an alcoholic beverage made from fermentation and distillation of the sap of coconut flowers) the previous day. Initial blood investigations (Haemoglobin $15.9 \mathrm{~g} / \mathrm{dl}$ ) and liver functions were normal. He was referred to a teaching hospital where he was treated for four days and, despite persistent retrosternal pain, discharged with a diagnosis of alcohol induced gastritis. He developed a small amount of haematemesisand was re-admitted on the same day. Chest X- ray and an upper GI endoscopy were not carried out. About 12 hours after admission, he developed massive haematemesis, for which he underwent emergency anterior gastrectomy. Three litres of blood was found in the stomach during surgery but no bleeders were identified. He 
succumbed, 30 min after admission to the intensive care unit.

Thoracic dissection at autopsy revealed ulceration $2 \mathrm{~cm}$ below the aortic arch with an impacted fish bone creating a fistula tract with the oesophagus (Figure 1). The fish bone was triangular in shape measuring $3.5 \times 2.5 \mathrm{~cm}$ with sharp pointed ends (Figure 2). The junction of the upper and middle third of the oesophagus was perforated and showed inflammation around the fish bone (Figure 3).There was clotted blood in the stomach. There were no oesophageal varices and liver did not show features of cirrhosis.

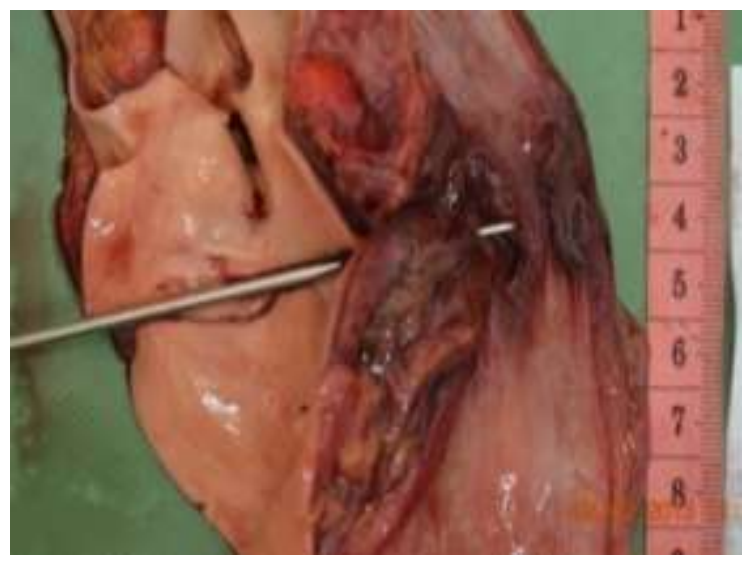

Figure1: Fistula tract

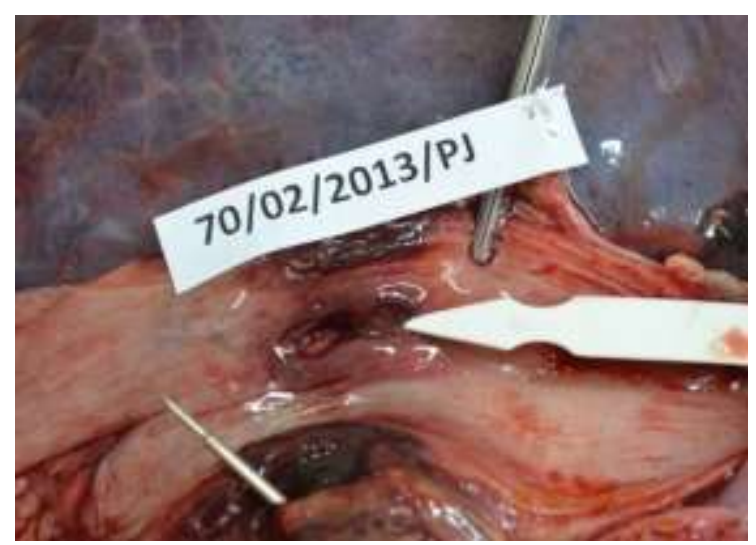

Figure 3: Oesophageal inflammation
There was no haemoperitoneum and sutures of the partial anterior gastrectomy were intact. Stomach mucosa was healthy and no features of gastritis were identified. Small and large intestines were filled with tarry stools. There were subendocardial flame shaped haemorrhages (Figure 4), and pale kidneys with prominent corticomedullary demarcation. All other internal organs were devoid of major pathologies. Histopathology of fixed tissues was unremarkable. Cause of death was haemorrhagic shock due ton aortooesophageal fistula caused by impaction of a fish bone in the oesophagus.

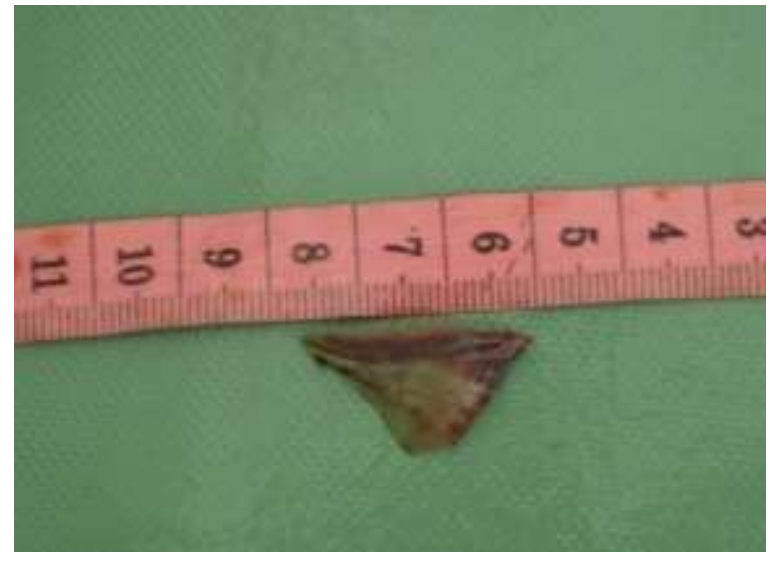

Figure 2: Fish bone

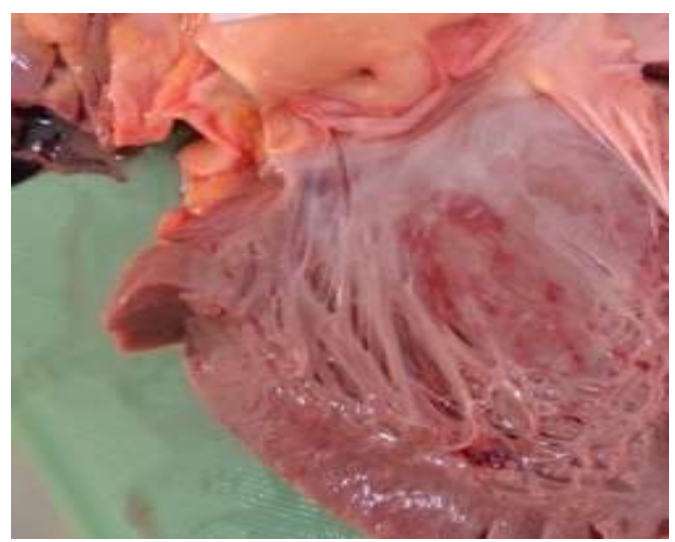

Figure 4: Subendocardial haemorrhage 


\section{Discussion}

Foreign body ingestion is a common presentation to a medical care facility. The majority of foreign bodies occur in the paediatric population with a peak incidence between ages 6 months and 6 years. ${ }^{[2]}$ However rarely neonates ${ }^{[3]}$ are also affected. In adults, true foreign body ingestion occurs more commonly in those with psychiatric disorders, development delay, alcohol intoxication, and individuals with secondary gain via release to a medical facility. ${ }^{[2]}$ This patient was a habitual alcoholic who had ingested fish while having arrack. Foreign bodies in the gastrointestinal (GI) tract can range from a coin to sharp objects including bone pieces, pins, needles, and tooth picks. Non sharp objects such as coins are more common in children, whereas, bone pieces are common in adults. $^{[4]}$ Due to passive and un-adapted peristalsis, oesophageal foreign bodies are the most common, and they tend to retain giving rise to complications compared with the other parts of the GI tract. ${ }^{[4]}$

Children with oesophageal foreign bodies are often asymptomatic. ${ }^{[5]}$ Adults will complain of odynophagia, persistent sensation of foreign body on swallowing, blood stained sputum and history of choking and gagging while eating. ${ }^{[4]}$ This particular patient presented to hospital complaining of something sticking retrosternally.

Nearly $80 \%$ of ingested foreign bodies pass without need for intervention. ${ }^{[6,7]}$ Less than $1 \%$ of foreign bodies cause perforation of the GI tract. ${ }^{[8]}$ Impaction, perforation, and obstruction occur at gastrointestinal narrowing's or angulations. ${ }^{[9]}$ In this case, the fish bone was found to be impacted at an angle in the oesophagus at autopsy. Ingestion of sharp objects increases the risk of perforation. ${ }^{[2]}$ In this case, the deceased had ingested a sharp, pointed fish bone which had impacted at the junction of upper and middle third of the oesophagus; a common site of impaction. ${ }^{[9]}$

Common causes of AEF are rupture of a thoracic aortic aneurysm, aortic dissection, foreign bodies, oesophageal cancer and thoracic surgery. ${ }^{[10]}$ Chiari described the aorto-oesophageal syndrome as a painful oesophageal injury followed by an asymptomatic interval, then signal haemorrhage followed by massive haematemesis hours or days later. ${ }^{[11]}$ In a Chinese study it was found that the average latent period between time of swallowing of the foreign body and the signal haemorrhage was about 8 days. ${ }^{[12]}$ In this case the patient had the signal haemorrhage 6 days after ingestion of fish. One review of all cases of AEF over a 30-year period found that mid thoracic pain, dysphagia, and sentinel arterial haemorrhage were common presentations. ${ }^{[10]}$ In this case the patient has had that typical triad of symptoms; mid thoracic pain, sentinel arterial haemorrhage, and exsanguination after an asymptomatic period of 12 hours.

Biplane radiographs identify most true foreign objects and free mediastinal or peritoneal air. ${ }^{[9]}$ Radiographs can confirm the location, size, shape, and number of foreign bodies. With suspected foreign body ingestion, persistent oesophageal symptoms should be evaluated by endoscopy, even in the setting of a negative radiographic evaluation. ${ }^{[9]}$ Diagnosis of AEF can be made with history alone, since the other causes of upper GI haemorrhage will not cause torrential bleeding as in AEF and the bright red arterial type of haemorrhage can distinguish it from variceal bleeding; the commonest cause of upper GI haemorrhage. ${ }^{[13]}$ Classic endoscopic finding is direct visualization of pulsatile blood. ${ }^{[13]}$ Aortography may be the best way of diagnosis of $\mathrm{AEF} \cdot{ }^{[13]} \mathrm{A}$ high index of suspicion coupled with endoscopy, CT scanning and aortography can assist in an early diagnosis, thereby, help in saving the life of the patient. ${ }^{[14]}$ In this case, neither X- ray nor endoscopy was done, even though the classical features were present.

Since the first case reported with AEF was described in 1818, there were no successfully managed cases till 1980. ${ }^{[15]}$ Mortality of AEF is around 40-60 \% [16].In one retrospective study, only three cases with AEF due to foreign body had survived, from a group of 32 patients between the year 1963 and 2010. ${ }^{[17]}$ Widely accepted approach for treating AEF caused by foreign body is a thoracotomy with aortic stent graft reposition followed by reconstruction of oesophagus. ${ }^{[18]}$ Use of a Sengstaken Blakemore tube as a temporary method to control bleeding prior to surgery is useful. ${ }^{[18,19]}$ Mortality of patients without surgical interventions is said to be higher comparatively to non-surgical methods. ${ }^{[17,18,20]}$ However, now it is recommended that 
combined surgical, minimally invasive procedures such as endovascular and oesophageal stent replacement will yield better results with minimal morbidity and mortality. ${ }^{[21,22,23]}$

In this patient the history was not followed by the essential investigations such as chest $\mathrm{X}$ - ray or upper GI endoscopy and the surgical team did not suspect or attempt to treat aorto-oesophageal fistula until massive bleeding took place. Therefore, we would also like to highlight the medico legal aspects of this case.

Medical negligence simply implies failure to exercise the degree of competence of an ordinary skilful doctor. ${ }^{[24]}$ Once a doctor-patient relationship is established, a legal relationship comes into existence which leaves the doctor open to an action of negligence by the aggrieved patient or next-of-kin To prove medical negligence, it is necessary to determine that there is a clear act of negligence and not mere "medical misadventure" that caused damage (injury or death) to the patient. ${ }^{[25]}$ The plaintiff must prove the four criteria against the particular institute or doctor to prove a case of medical negligence. Firstly, there has to be an established doctor patient relationship. This patient has presented himself to a government hospital and a doctor patient relationship was established. Secondly, it should be proved that the doctor failed to give a reasonable degree of duty of care to the patient; in this case the primary care doctor has fulfilled his duty by transferring the patient to a teaching hospital where X-ray and endoscopy facilities were available. However, despite the history of fish ingestion, tertiary care doctors have not suspected foreign body ingestion, for which an X-ray or an esophagogastroduodenoscopy is recommended. And also the surgeons have not thought of the possibility of aorto-oesophageal fistula during the laparotomy. Thirdly the plaintiff must prove that the act of negligence caused damage to the patient. In this instance a tragic death occurred due to omission of acts. And finally it must be proved that the death did not occur due to a cause beyond the control of the doctor, but purely due to doctor's failure to give a reasonable degree and duty of care to the patient. ${ }^{[24,26]}$ Here it may be argued that even if they diagnosed aorto-oesophageal fistula earlier the patient may not survive, since it carries a high mortality rate. However, it is up to the court to decide whether it is a reasonable excuse to escape alleged medical negligence, since there are cases of survival with advanced treatment as mentioned earlier. We presented our case in order to increase the awareness of the dreaded complications of foreign body ingestion to the public as well as to the primary care doctors, surgeons, and emergency departments and to highlight the fact that medical negligence can be alleged even in a rare case.

\section{References}

1. Marone EM, Coppi G, Kahlberg A et al. Combined endovascular and surgical treatment of primary aortoesophageal fistula. Texas Heart Institute Journal.2010:37;722-724

2. Ikenberry SO, Jue TL, Anderson MA, et al. Management of ingested foreign bodies and food impactions. Gastrointestinal Endoscopy 2011;73:1085-91

3. Singh R, Pandit C, Gupta D, Vajifdar H. Foreign body esophagus in a neonate: Unusual age and unusual presentation. Journal of Anaesthesiology Clinical Pharmacology 2012;28:258.

4. Nandi P, Ong GB. Foreign body in the esophagus: Review of 2394 cases. British Journal of Surgery 1978; 65:5-9

5. Arana A, Hauser B, Hachimi-Idrissi S, Vandenplas Y. Management of ingested foreign bodies in childhood and review of the literature. European Journal of Pediatrics 2001; 160:468-72

6. Carp L. Foreign bodies in the Intestine. Annals of Surgery 1927; 85:575-591

7. Pellerin D, Fortier-Beaulieu M, Gueguen J. The fate of swallowed foreign bodies experience of 1250 instances of sub-diaphragmatic foreign bodies in children. Progress in Paediatric Radiology. 1969; 2:286-302.

8. Hunter TB, Taljanovic MS. Foreign Bodies. Radiographics 2003;23:731-57

9. Ginsberg GG. Management of ingested foreign objects and food bolus impactions. Gastrointestinal Endoscopy 1995;41:33-8 
10. Hollander JE, Quick G. Aorto-esophageal Fistula: A comprehensive review of the literature. The American Journal of Medicine 1991; 91:279-87.

11. Sloop RD, Thompson JC, Aorto-oesophageal fistula: report of a case and review of literature. Gastroenterology 1967;53:768-77

12. Li S. Aorto-esophageal fistula caused by swallowed foreign body (report of 17 cases). Zhonghua Er Bi Yan Hou Ke Za Zhi. 1992;27(2):91-2

13. Heckstall RL, Hollander JE. Aortoesophageal Fistula: Recognition and Diagnosis in the Emergency Department. Annals of Emergency Medicine 1998;32:502-5

14. Nawaf JS, Kamal E.B. Ingested chicken bone leading to aorto esophageal fistula. Saudi Med J 2005; 26:1442-1444

15. Ctercteko G, Mok CK. Aorto-esophageal fistula induced by a foreign body: the first recorded survival. Journal of Thoracic and Cardiovascular Surgery 1980;80:233-235

16. Gobolos L, Miskolczi S, Pousios D, et al. Management options for aorto-oesophageal fistula: case histories and review of the literature. Perfusion 2013;28:286-90

17. Zhang X, Liu J, Li J, et al. Diagnosis and treatment of 32 cases with aortoesophageal fistula due to oesophageal foreign body. The Laryngoscope 2011; 121:267-72.

18. Yamada T, Sato H, Seki M, et al. Successful salvage of aortoesophageal fistula caused by a fish bone. Annals of Thoracic Surgery 1996;61:1843-5

19. Mccomas BC, Miles PV, Katz BE. Successful salvage of an 8-month-old child with an aortoesophageal fistula. Journal of Pediatric Surgery 1991; 26:1394-5.

20. Kubota S, Shiiya N, Shingu Y, et al. Surgical strategy for aorto-esophageal fistula in the endovascular era. General Thoracic and Cardiovascular Surgery 2013;61:560-4

21. Chen X, Li J, Chen J, et al. A combined minimally invasive approach for the treatment of aorto-oesophageal fistula caused by the ingestion of a chicken bone: case report and literature review. Clinics 2012; 67:195-7.

22. Kelly SL, Peters P, Ogg MJ, et al. Successful management of an aorto-esophageal fistula caused by a fish bone - case report and review of literature. Journal of Cardiothoracic Surgery 2009;4:21

23. Baril DT, Carroccio A, Ellozy SH, et al. Evolving strategies for the treatment of aortoenteric fistulas. Journal of Vascular Surgery 2006; $44: 250-7$.

24. Mason JK, McCall Smith RA, Laurie GT. Law and medical ethics. $6^{\text {th }}$ ed. London: Reed Elsevier;2003:9.16 - 9.24

25. Fernando R, De Silva LC. Basic principles of medical negligence. Colombo: Institute of Health Studies; 1996:8

26. De Alwis LBL. Lecture notes in Forensic Medicine. $1^{\text {st }}$ ed. Colombo: Primal; 2011;182 